\title{
Le raisonnement clinique: données issues de la recherche et implications pour l'enseignement
}

\author{
Clinical reasoning: from research findings to applications for teaching
}

Mathieu NENDAZ', Bernard CHARLIN², Vicki LEBLANC ${ }^{3}$, Georges BORDAGE ${ }^{4}$

\section{Messages clés}

- Il existe un vaste champ de connaissances en psychologie cognitive appliquée à l'éducation médicale. Ces connaissances ont permis de mieux comprendre les processus du raisonnement, l'organisation des connaissances et le recueil de linformation clinique et de développer certaines stratégies pédagogiques fondées sur des données probantes. $\bullet$ Les médecins font appel à des processus mixtes de raisonnement, associant des stratégies analytiques, comme le raisonnement hypothético-déductif, et des stratégies non analytiques telle la reconnaissance spontanée d'une conjonction de signes cliniques. - L'organisation des connaissances peut prendre diverses formes dont les plus élaborées consistent en des réseaux richement interconnectés. - L'activation des connaissances passe par le plus souvent une étape de représentation mentale de la teneur du problème clinique. - L'acquisition des données cliniques est conditionnée par la génération précoce d'hypothèses diagnostiques. - L'apprentissage des seuls processus de raisonnement n'est pas efficace sil ne s'accompagne pas de l'acquisition simultanée des connaissances spécifiques nécessaires pour résoudre un problème clinique.

Mot clés Raisonnement clinique; éducation médicale; compétence clinique; revue de la littérature.

\section{Key Messages}

- Research in cognitive psychology has led to the development of several theories and models of clinical reasoning, knowledge organization and activation, and the acquisition of relevant clinical data. - There is no unique process of reasoning because of the close interrelationship between reasoning and knowledge, thus leading physicians to use both analytical and non-analytical processes. - Knowledge can be organized in many different ways in memory, the most elaborated of which contains rich networks of interconnected knowledge. - Initial problem representation facilitates the activation of knowledge in memory. - The acquisition of clinical data is most often led by the early generation of diagnostic hypotheses. - Teaching reasoning processes in isolation of knowledge acquisition is doomed to fail because of the close interrelationship between reasoning and knowledge.

Key words Clinical reasoning; medical education; clinical competence; literature review.

Pédagogie Médicale 2005; 6: 235-254

1- Service de médecine interne générale et Unité de recherche et de développement en éducation médicale - Faculté de médecine - Université de Genève - Suisse.

2- Unité de recherche et développement en éducation des sciences de la santé - Faculté de médecine - Université de Montréal - Québec - Canada

3- Wilson Centre for Research in Education - Faculty of Medicine - University of Toronto

4- Department of Medical Education - College of Medicine - University of Illinois at Chicago

Correspondance: Mathieu Nendaz - Service de médecine interne générale - Hôpitaux Universitaires - 1211 Genève 14

Téléphone: +4122 3729059 - Télécopie: +4122 3729116 - mailto: mathieu.nendaz@hcuge.ch 


\section{Références}

\section{Introduction}

On nomme raisonnement clinique les processus de pensée et de prise de décision qui permettent au clinicien de prendre les actions les plus appropriées dans un contexte spécifique de résolution de problème de santé ${ }^{1,2}$. Il peut être considéré comme l'activité intellectuelle par laquelle le clinicien synthétise l'information obtenue dans une situation clinique, l'intègre avec les connaissances et les expériences antérieures et les utilise pour prendre des décisions de diagnostic et de prise en charge ${ }^{3}$. On le désigne souvent sous le terme de résolution de problème clinique.

Ces processus de pensée et de prise de décision sont au cour de l'exercice professionnel. Ils constituent un phénomène hautement complexe. Aucune théorie ni aucun modèle unique issu de données de recherche ne sont acceptés par tous ${ }^{1,4}$. Pour enseigner et évaluer efficacement le raisonnement dans un domaine, il est pourtant essentiel d'avoir une perception claire des processus qui le caractérisent ${ }^{5}$. L'étendue de la littérature et la diversité des approches rendent difficile l'appropriation des concepts du raisonnement clinique. Cet article présente les données issues de la recherche, en ayant explicitement comme fil conducteur l'intention de proposer une synthèse utile aux enseignants des sciences de la santé désireux de mieux enseigner et mieux évaluer le raisonnement clinique. L'exhaustivité étant impossible dans une telle entreprise, nous avons fait délibérément le choix, au sein des multiples études réalisées, d'en sélectionner certaines qui nous semblent avoir des implications pour les éducateurs. Le texte ne contient pas de détails sur les méthodes de recherche qui ont conduit aux résultats décrits. Les lecteurs intéressés sont invités à les approfondir à partir des références présentées. La perspective fait résolument référence à la psychologie cognitive car cette discipline représente le courant dominant d'influence en éducation des sciences de la santé $^{6-8}$. Soulignons enfin que l'étude du raisonnement clinique peut être abordée selon deux grandes approches. La première, descriptive, vise à révéler les processus utilisés naturellement par les cliniciens. C'est l'approche de la psychologie cognitive. La deuxième, appelée analyse décisionnelle, est prescriptive et vise à optimiser le raisonnement clinique, notamment par une approche probabiliste ${ }^{9}$. Le présent article de synthèse ne concerne, à l'exception de l'approche bayésienne, que la première approche.

\section{Les processus de raisonnement}

Les divers travaux de recherche menés à ce sujet ont révélé l'existence de plusieurs processus de raisonnement clinique qui peuvent être distingués en processus analytiques et non analytiques.

\section{Les processus analytiques \\ Le processus hypothético-déductif}

En 1978, paraissait une monographie rassemblant plusieurs études menées à l'Université de l'état du Michigan et portant sur les processus de résolution de problèmes médicaux ${ }^{10}$. Ces études, réalisées par Elstein et coll., s'appuyaient sur des méthodes de recherche particulièrement innovatrices pour l'époque, permettant d'analyser le raisonnement dans des conditions contrôlées s'approchant le plus possible de la pratique. Les méthodes faisaient appel à l'observation directe de médecins en situation de résolution de problèmes cliniques en présence de patients, à l'enregistrement du raisonnement à voix haute, à l'analyse de protocoles de pensée à voix haute et à la comparaison systématique entre novices et experts. Ces travaux ont eu un impact majeur au sein des communautés de chercheurs en psychologie et en éducation des sciences de la santé et sont toujours d'actualité plusieurs années plus tard ${ }^{11}$.

Ces études démontraient que des hypothèses diagnostiques (définies par les auteurs comme des solutions entrevues au problème posé) apparaissent très tôt à l'esprit du clinicien, dès les premières minutes de l'entrevue médicale, à partir de la plainte principale du malade, de quelques signes et du contexte perçu. Le nombre d'hypothèses émises est limité (rarement plus de 4 ou 5 ) et elles servent de cadre au recueil d'informations supplémentaires. Les données cliniques sont interprétées en permanence pour juger si elles sont compatibles avec l'hypothèse testée. Puis l'hypothèse est elle-même évaluée pour être soit retenue, soit écartée, soit vérifiée par une recherche orientée de données complémentaires. Ce processus, appelé hypothético-déductif (Figure 1), recommence avec de nouvelles hypothèses, jusqu'à ce que le médecin atteigne le diagnostic de présomption. Les auteurs notaient également que le processus de génération précoce d'hypothèses est irrépressible. Il survient même lorsque les cliniciens reçoivent l'instruction formelle de s'en abstenir. Une autre étude révèle d'ailleurs que lorsque la consigne formelle de s'abstenir 


\section{Figure 1 : \\ Représentation du processus hypothético-déductif du raisonnement clinique}

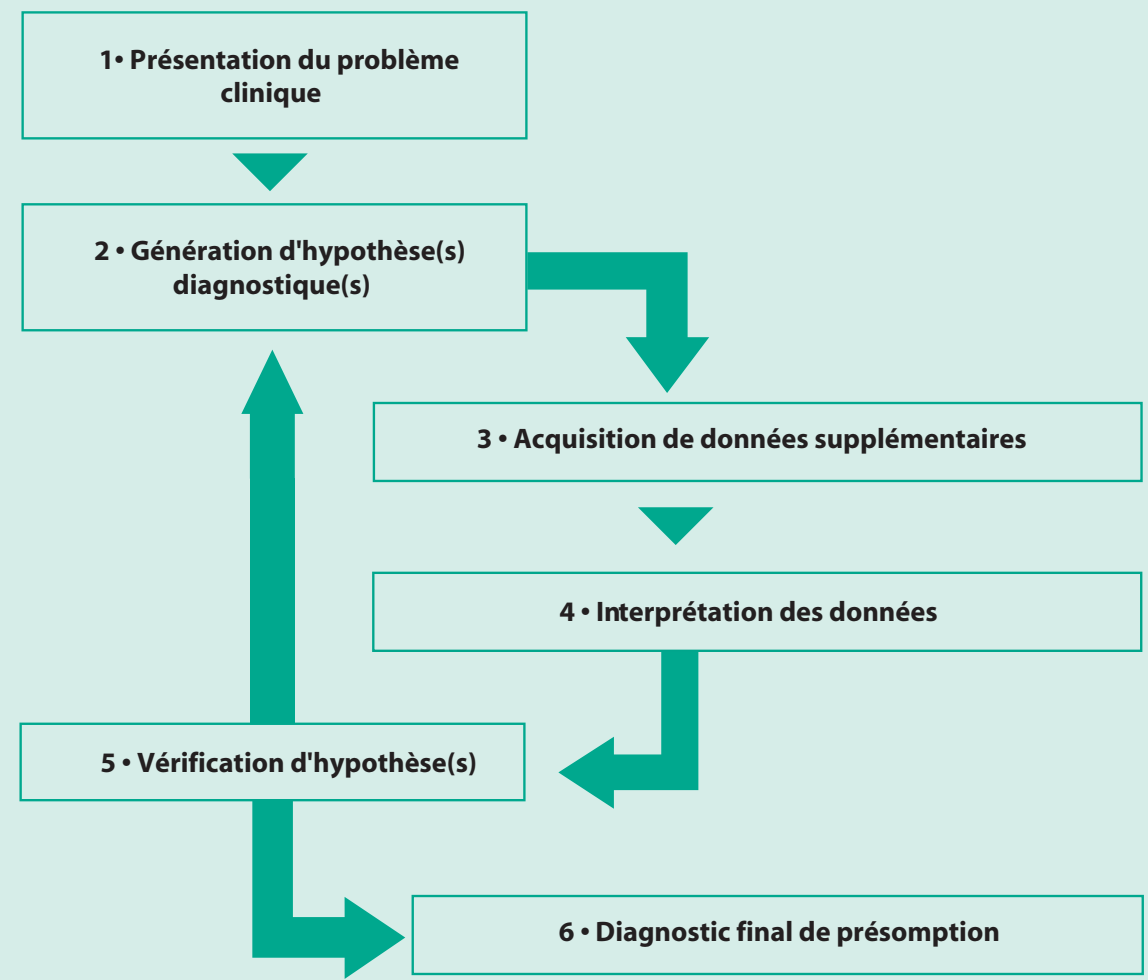

d'une génération précoce d'hypothèses est respectée chez des novices, ceci mène à une augmentation du nombre d'erreurs au niveau de l'interprétation des données comme à celui des conclusions diagnostiques ${ }^{12}$. Selon Elstein et coll., la génération d'hypothèses est une nécessité psychologique, compte tenu de la complexité des situations cliniques, de l'énorme quantité de données disponibles chez le patient et de la capacité limitée de la mémoire de travail (aussi nommée " mémoire à court terme »). La génération d'un nombre limité de solutions possibles est un moyen efficace de transformation d'un problème mal structuré en un problème mieux structuré. L'omniprésence du processus hypothético-déductif a ensuite été largement confirmée par d'autres chercheurs ${ }^{13-16}$.

Dans le processus hypothético-déductif, une solution possible au problème est envisagée (l'hypothèse) et une démarche analytique délibérée survient par activation d'un réseau de connaissances associant les hypothèses et leurs signes cliniques, avec recherche active des signes positifs ou négatifs qui permettent de les confirmer ou infirmer. Ce processus, qui va de la solution entrevue vers les données (chaînage arrière, "backward reasoning " en anglais), implique une démarche analytique et consciente sur le mode déductif.

\section{Lapplication de règles causales ou conditionnelles}

Un autre processus de raisonnement analytique consiste à cheminer consciemment des données vers la solution (chaînage avant, "forward reasoning " en anglais). Dans des études portant sur l'analyse syntaxique du raisonnement à voix haute de médecins confrontés à des cas cliniques complexes décrits sur papier, Patel et coll. ${ }^{17-19}$ ont affirmé que les cliniciens experts se distinguent des cliniciens novices par l'absence d'utilisation du raisonnement hypothético-déductif. Selon ces auteurs, les experts raisonnent en suivant des règles causales ou conditionnelles (si le symptôme $X$ est présent... alors cela implique le diagnostic $Y . .$. ). Le raisonnement consiste donc en un processus conscient et analytique dans lequel les méde- 


\section{Références}

cins reconnaissent un ensemble de données critiques et appliquent des règles stockées en mémoire. Le processus part des données cliniques et aboutit au diagnostic, ce qui est à l'opposé du processus hypothético-déductif qui part des hypothèses pour chercher des données pertinentes. Par exemple, "si il y a un ictère, des angiomes stellaires, un érythème palmaire, une hypertrophie parotidienne bilatérale, une circulation collatérale (...), alors il s'agit d'une cirrhose avec insuffisance hépato-cellulaire d'origine éthylique ". Ces affirmations ont été critiquées par Elstein ${ }^{11}$ et par Eva $^{20}$ comme étant le résultat d'artéfacts d'interprétation liés aux tâches données aux cliniciens étudiés, en l'occurrence un travail d'analyse de cas complexes, multifactoriels, pour lesquels aucune hypothèse simple ne peut être émise d'emblée. Par ailleurs, dans la méthode utilisée, l'ensemble des données cliniques est d'emblée soumis aux sujets, ce qui peut influencer le déroulement du raisonnement ${ }^{21}$. Le modèle proposé n'explique donc pas comment, dans une démarche clinique réelle, le clinicien s'y prend pour aller chercher l'information complémentaire qui va l'aider dans sa progression vers la solution. Ce processus de raisonnement analytique et conscient est sans doute utilisé lorsque les cliniciens ne perçoivent pas d'hypothèse pertinente dans un tableau clinique complexe.

\section{Lapproche bayésienne}

En plus de ces processus décrits essentiellement dans des travaux conduits par des psychologues de la cognition, il faut encore évoquer un modèle analytique de raisonnement qui fait appel au théorème de Bayes ${ }^{22}$. Selon ce modèle, devant une présentation de cas, le clinicien a une conception a priori de la probabilité d'un diagnostic particulier et des probabilités conditionnelles associant chaque élément complémentaire (les signes, les données des tests diagnostiques) au diagnostic envisagé. Chaque élément complémentaire incorporé au problème conduit à un calcul de la nouvelle probabilité, a posteriori, du diagnostic envisagé. Ce processus, proche des conceptions de la médecine fondée sur les données probantes 9 , a été introduit dans une approche pédagogique visant à aider la démarche diagnostique des étudiants, en leur fournissant une description explicite des relations de probabilité entre signes et maladie ${ }^{23}$. Il ne s'agit cependant pas là d'un appel à des processus de raisonnement utilisés naturellement par les cliniciens, mais d'une méthode prescriptive qui vise à optimiser le raisonnement par des méthodes proches de celles de systèmes informatiques experts. Il est intéressant de noter qu'en matière de résolution de problèmes cliniques, les comparaisons avec des systèmes experts informatisés révèlent que la performance des médecins est régulièrement au moins aussi bonne que celle des ordinateurs, alors que ces derniers possèdent des avantages évidents en terme de fiabilité de mémoire et de vitesse de traitement de l'information. Les experts doivent donc avoir de meilleures représentations des connaissances, de meilleures méthodes d'inférences, ou les deux, sachant qu'ils sont désavantagés sur le plan de l'architecture et de l'accès en mémoire ${ }^{24}$.

\section{Les processus non analytiques}

Le deuxième groupe de processus concerne les processus non analytiques, c'est-à-dire inconscients et automatiques. Ils consistent à identifier au sein d'un cas clinique soit des configurations caractéristiques de signes (pattern recognition ${ }^{25,26}$, soit une similarité avec des cas rencontrés précédemment ${ }^{27}$. Dans les deux cas, l'acte de catégorisation repose sur un jugement à propos de la qualité de la similarité entre le cas présent et une configuration de signes, une image ou un cas stockés en mémoire. Ces processus se développent surtout dans certaines situations non problématiques et familières aux cliniciens, de façon automatique. Selon le modèle dit de cas concrets (instances) ${ }^{28}$, les cliniciens possèdent un répertoire de cas rencontrés précédemment et, lorsqu'ils se trouvent face à un nouveau patient, ils comparent la présentation clinique à celles stockées en mémoire pour l'associer à un diagnostic donné. Ces cas concrets sont stockés tels qu'ils ont été vécus, sans abstraction, et l'apprentissage consiste en l'addition de nouvelles expériences cliniques à la base de données en mémoire à long terme. Selon ce modèle, l'utilisation de processus non analytiques serait inexistante chez des purs novices, puisqu'ils n'ont pas d'expériences passées à appeler en mémoire, mais augmenterait avec l'expérience clinique. En revanche, même des étudiants en médecine peuvent faire appel à des processus non analytiques en générant ainsi des hypothèses diagnostiques d'emblée ${ }^{20}$. Le stade le plus élevé de la compétence médicale se caractérise ainsi par l'existence en mémoire d'un énorme répertoire de cas concrets et le raisonnement à ce stade n'est pas réellement une résolution de problème, mais un repérage en mémoire de solutions "toutes faites " car antérieurement déjà mises en oeuvre ${ }^{7}$. 
L'existence de tels processus a été démontrée par Brooks et coll. dans une série d'études menées dans des domaines où les informations de nature visuelle sont prépondérantes (par exemple en dermatologie ou lorsque les cas comportent des signes physiques évidents). Par exemple, si l'on fait précéder des tâches diagnostiques par une présentation de cas, la justesse diagnostique est supérieure pour les cas visuellement similaires par rapport aux cas perceptuellement distincts ${ }^{29}$. De plus, Hatala et coll. ${ }^{30}$ ont montré que même des éléments non pertinents pour le diagnostic (par exemple, le fait d'être banquier) ont un impact sur la performance diagnostique pour des cas présentés ultérieurement, dans lesquels l'élément non pertinent est introduit. L'interaction de processus analytiques et non analytiques au sein du raisonnement clinique doit être soulignée. Par exemple, donner une suggestion diagnostique décroît la perception ${ }^{31}$ et le poids relatif ${ }^{32}$ des signes qui orientent vers des alternatives diagnostiques.

\section{Les processus mixtes (analytiques et non analytiques)}

Les travaux décrits ci-dessus démontrent indiscutablement l'existence de jugements de similarité dans les processus de raisonnement diagnostique. Mais ces jugements résument-ils le processus? Nombre d'éducateurs cliniciens ${ }^{33}$ jugent dangereux un processus diagnostique qui s'appuierait exclusivement sur un jugement non analytique de similarité (ce cas me rappelle le cas de Mme $X$ qui avait le diagnostic $Y$; donc ce cas est $Y$ ). En revanche, la reconnaissance d'une similarité est sans doute le moyen d'activation en mémoire d'une solution (une hypothèse) et du réseau de connaissances relatif à cette hypothèse. Cette première étape d'activation est alors suivie d'une étape de confirmation sur le mode hypothético-déductif. En dermatologie par exemple, le raisonnement est souvent: l'aspect évoque un cancer baso-cellulaire (jugement de similarité). S'il s'agit bien de cela, je devrais trouver en périphérie un aspect perlé et des télangiectasies (confirmation sur le mode hypothéticodéductif). L'observation non formelle des cliniciens révèle que cette combinaison "reconnaissance de similarité - confirmation hypothético-déductive " est très courante. Formellement, ceci a été démontré par Kulatunga-Moruzi et $\operatorname{coll}^{34,35}$. Ces chercheurs ont démontré que la performance diagnostique d'étudiants, résidents et cliniciens est affectée si l'on modifie la similarité des cas cliniques (processus non analytique) et la typicité des cas (processus analytique). En plus, des instructions concernant le processus à utiliser affectent aussi la performance. Les participants qui sont incités à utiliser un processus basé sur la reconnaissance de similarité suivi d'un processus basé sur l'analyse des règles et des signes ont une performance supérieure à ceux qui sont encouragés à ne suivre qu'un seul des deux processus.

D'autres travaux illustrent le fait que les processus de perception automatique et de traitement analytique de l'information s'influencent mutuellement. Lorsqu'on présente à des médecins expérimentés une série de courtes histoires cliniques sans autre donnée (par exemple, homme de 20 ans avec asthénie), la performance diagnostique de base est d'environ $20 \%$. Si on leur montre en plus une photo du patient contenant des signes physiques compatibles avec le diagnostic (exemple: ptose palpébrale), de manière non surprenante, la performance diagnostique augmente à environ $60 \%$. De façon intéressante, lorsqu'on indique au médecin ce qu'il faut voir sur la photo (exemple: il y a une ptose palpébrale), la performance diagnostique augmente encore (jusqu'à $80 \%$ ), ce qui signifie qu'il existe un type d'erreur consistant à ne pas voir des éléments importants, pourtant accessibles, ou bien à les remarquer, mais sans savoir les interpréter et les intégrer. Inversement, les données clés sont mieux reconnues quand on suggère le bon diagnostic au médecin (par exemple: myasthénie grave). Cette étude ${ }^{36}$ et celles de LeBlanc ${ }^{37,} 38$ démontrent que la qualité du diagnostic considéré précocement par le clinicien est associée à la reconnaissance des signes cliniques pertinents d'un cas. $\mathrm{Si}$ le diagnostic considéré est correct, leur reconnaissance sera plus précise alors que s'il est erroné, des erreurs seront commises. Des signes présents ne seront pas reconnus et des signes pourtant absents seront perçus.

Nous verrons dans la section concernant les applications pratiques pour les enseignants comment tenir compte de la coexistence de processus analytiques et non analytiques dans le raisonnement médical ${ }^{39}$.

\section{L'organisation des connaissances}

L'étude des processus de raisonnement n'explique cependant pas la capacité à résoudre adéquatement les problèmes cliniques. En effet, un processus analytique de raisonnement avec génération précoce d'hypothèses peut être utilisé tant par les cliniciens qui parviennent au diagnostic que par ceux qui n'y parviennent pas ${ }^{15}$. Il faut dès lors expliquer l'expertise d'une autre manière. 


\section{Références}

Les chercheurs se sont orientés vers l'étude de l'organisation des connaissances cliniques mobilisées pendant le développement du processus de raisonnement clinique. Leurs travaux ont conduit à considérer que l'acquisition de l'expertise est caractérisée par le développement en mémoire de représentations cognitives, notion qui rend compte de l'architecture des connaissances, conséquence de l'ensemble des connexions et des liens établis entre concepts et/ou expériences vécues (la mémoire des cas rencontrés) ${ }^{7}$. Ces liens unissant concepts et expériences sont porteurs de sens et la compétence dans un domaine se caractérise par l'existence de réseaux élaborés ${ }^{40}$. Le terme " élaboré " traduit la présence de liens qui structurent et connectent les éléments de connaissances, incluant la force de leurs associations. La maîtrise d'un domaine se définit non pas simplement par la quantité d'information qu'une personne possède, mais par la façon dont cette information est organisée. Il est intéressant de noter que les réseaux ne sont pas identiques d'un clinicien à l'autre et ce, même si les niveaux d'expérience clinique sont similaires ${ }^{41}$ et qu'ils peuvent différer chez un même individu selon le contexte dans lequel les concepts sont présentés ${ }^{7,42}$.

En dépit de ses particularités individuelles, l'organisation des connaissances en mémoire permet aux cliniciens expérimentés de traiter efficacement l'information dans leurs tâches cliniques ${ }^{7,43}$. Les signes cliniques d'une situation activent des connaissances, des concepts ou des exemples similaires au sein du réseau cognitif, qui à leur tour en appellent d'autres. Ce processus d'activation s'étend, de sorte que, très rapidement, le clinicien dispose d'un riche ensemble de connaissances adaptées à la situation clinique, ce qui lui permet de générer des hypothèses pertinentes et de rechercher des informations supplémentaires pour les confirmer ou infirmer. Selon cette conception, l'acte diagnostique d'un expert ne résulte pas de l'application d'une stratégie générale de résolution de problème, mais de l'activation en mémoire de réseaux de connaissances appropriés. Ces représentations cognitives peuvent être organisées selon plusieurs modèles ${ }^{44}$.

\section{Les architectures fondées sur les exemples concrets (instances)}

Les connaissances résultent de l'accumulation en mémoire de cas cliniques tels qu'ils ont été vécus, sans processus d'abstraction. Chaque caractéristique clinique mémorisée garde donc une structure indépendante, non modifiée. L'apprentissage correspond donc à l'addition de nouveaux exemplaires pour former une collection de cas, utilisée dans le processus diagnostique pour reconnaitre des nouvelles situations cliniques similaires (processus non analytiques décrits ci-dessus). Des éléments contextuels du cas peuvent également être mémorisés (par exemple, le fait que le patient ait séjourné en pays tropical ou qu'il soit menuisier), ce qui reflète l'importance du contexte dans le processus rapide de reconnaissance immédiate d'un cas, mais également dans la génération d'erreurs diagnostiques potentielles ${ }^{45}$.

\section{Les architectures prototypiques}

Dans ce modèle, les caractéristiques des cas rencontrés ne sont pas mémorisées telles quelles mais subissent un certain degré d'abstraction dont la constellation peut aboutir à un ensemble de caractéristiques décontextualisées, une description sommaire du cas ou un exemple idéal du problème. Nous décrivons ici une théorie des prototypes plus particulièrement étudiée en médecine ${ }^{46}$. Pour que puisse s'initier un raisonnement clinique, les symptômes et signes présents chez un patient doivent évoquer des diagnostics ou des catégories diagnostiques correspondants. Selon cette approche, les catégories sont formées de prototypes, qui représentent l'abstraction d'un exemple typique d'une catégorie. Ainsi, la dissection aortique pourrait représenter un prototype illustrant une douleur thoracique irradiant vers le dos, ou l'infarctus du myocarde représenter un prototype de la maladie coronarienne en général. Tous les membres d'une catégorie ne sont pas considérés comme égaux. Ceux qui partagent plus de signes avec le prototype sont perçus comme étant des membres plus typiques d'une catégorie $^{46}$. Les cliniciens à qui on demande d'énumérer toutes les pathologies qui composent une catégorie de problèmes de santé tendent à énumérer les plus typiques en premier, c'est-à-dire celles qui ont le plus de signes en commun avec les autres membres de la catégorie. Lorsqu'on leur demande de vérifier l'appartenance d'une pathologie à une catégorie, les cliniciens y réussissent plus rapidement et avec moins d'erreurs pour les entités typiques (par exemple, angine de poitrine) que pour les entités moins typiques (par exemple, vasculite coronarienne) de chaque catégorie. Ceci est illustré par le fait que les médecins ont une meilleure compétence diagnostique lorsque le cas est typique que lorsqu'il ne l'est pas ${ }^{47}$. Ces prototypes sont importants pour l'enseignement, car ils servent de point d'ancrage à l'acquisition de nouvelles 
connaissances, de manière à former un grand réseau de connections entre prototypes et entre catégories.

On peut noter ici que les modèles bayésiens, qui reposent sur le lien de probabilité reliant les caractéristiques des patients et les catégories, constituent un exemple particulier d'une telle architecture prototypique.

\section{Les architectures complexes}

\section{Les réseaux sémantiques}

À partir de méthodes de la sémantique structurale, Lemieux et Bordage ${ }^{48}$ ont montré que les cliniciens analysent le langage spontané des patients pour en extraire la signification et lui donner un sens médical. En étudiant le discours de cliniciens raisonnant à voix haute, ils ont déterminé qu'ils transforment les données émises par le patient en formes s'agençant en opposition binaire, appelée axes sémantiques (par exemple continu/discontinu, aigu/chronique, moteur/sensitif). Cette transformation sémantique permet au clinicien de se bâtir une représentation du problème, de lui donner une signification, puis de comparer et contraster les hypothèses diagnostiques de façon à déterminer les relations cohérentes entre ces concepts et à activer les représentations cognitives pertinentes (réseaux, scripts, etc.) qui lui permettront finalement de s'orienter efficacement vers une solution diagnostique du problème clinique (Figure 2).

Ces réseaux sémantiques peuvent être plus ou moins développés. Bordage propose une classification du discours des médecins en quatre catégories, selon la richesse de l'organisation de leurs connaissances et leur capacité à comparer et contraster les hypothèses diagnostiques en utilisant les données cliniques (Tableau 1) $)^{40,49}$.

Les connaissances peuvent être:

1) réduites (le discours ne révèle ni effort de transformation sémantique, ni connexion entre données du patient et connaissances);

2) dispersées (quelques transformations, mais les hypothèses sont désordonnées, ne se réferent pas aux données obtenues et sont listées sans être comparées et contrastées les unes aux autres);

3) élaborées (les transformations sémantiques sont nombreuses et utilisées à bon escient pour comparer et contraster les hypothèses) ;

4) compilées (le clinicien reconnaît d'emblée un ensemble sémantique de données cliniques qu'il associe à une hypothèse clinique).

\section{Les scripts}

En sciences cognitives, on nomme scripts les architectures de connaissance adaptées à des actions spécifiques ${ }^{50,51}$. Ce concept a été transposé en médecine par plusieurs auteurs $^{28,43,52}$. Les conceptions different légèrement selon les auteurs mais l'essentiel de la théorie implique que les cliniciens possèdent des connaissances spécifiquement organisées pour être efficaces dans leurs tâches cliniques. On peut ainsi parler de scripts diagnostiques, de scripts d'investigation ou de scripts thérapeutiques, le tout étant regroupé sous le terme générique de scripts cliniques. Dans la perspective de la classification de Bordage exposée ci-dessus, un bon clinicien possèderait des scripts cliniques adaptés à son domaine de compétence: il possède donc des connaissances élaborées ou compilées.

La théorie des scripts postule que, devant un nouveau cas, les médecins mobilisent ces réseaux pertinents préétablis et les utilisent pour comprendre la situation et agir en fonction de leurs buts cliniques (diagnostiques, d'investigation ou de traitement). Les scripts diagnostiques contiennent les associations que le clinicien a établies entre l'entité pathologique, ses différents attributs cliniques (les signes et symptômes) et des cas similaires rencontrés précédemment. Ce sont ces associations et ces liens qui, dans le contexte clinique, permettent de prendre les décisions concernant la force ou la faiblesse d'une hypothèse ou de décider que tel signe ou symptôme n'est jamais associé à une maladie et donc que cette hypothèse doit être éliminée. Des liens de même nature permettent de raisonner pour aboutir aux décisions d'investigation ou de traitement.

Au début de la formation clinique, un novice n'a que quelques concepts sur les maladies d'un domaine et les liens sont lâches. Son raisonnement s'effectue surtout sur un mode physiopathologique en utilisant le savoir biomédical. Toutefois, les premières expériences cliniques, avec leurs contraintes de temps et d'efficacité, amènent l'étudiant à découvrir rapidement que le raisonnement sur un mode de relations causales (si tel organe est atteint, tel symptôme devrait être présent) est exigeant cognitivement. Il commence alors à utiliser les liens unissant signes et maladies et à bâtir ses premiers scripts cliniques qui lui permettent de raisonner sur un mode associatif (utilisation de la force des liens positifs ou négatifs unissant signes et maladies). L'utilisation de ces réseaux de connaissances est très efficace dans les tâches cliniques, parce que: 1) leur activation est auto- 


\section{Références}

\section{Tableau 1 : \\ Types de discours reflétant l'organisation de la pensée selon le modèle de réseaux sémantiques \\ (adapté de Bordage ${ }^{40,49}$ )}

Cas : M. B., 73 ans, se plaignant de fourmillements et de faiblesse de la main droite depuis 4 mois et de la main gauche depuis 2 mois. [...] L'examen physique révèle une atrophie des muscles intrinsèques de la main droite avec faiblesse à l'abduction des doigts et diminution de la sensibilité des $4^{\circ}$ et $5^{\circ}$ doigts, et une absence du réflexe ostéo-tendineux brachioradial droit. [...] Aux membres inférieurs, réflexes ostéotendineux vifs et symétriques avec clonus non soutenu des deux pieds [...].

\begin{tabular}{|c|c|c|}
\hline Type de discours & Description & Exemple \\
\hline Réduit & $\begin{array}{l}\text { Il n'y a aucun effort d'abstrac- } \\
\text { tion, pas de connections entre } \\
\text { les données du patient et les } \\
\text { connaissances. }\end{array}$ & $\begin{array}{l}\text { Je ne sais pas. Je ne me souviens pas de ce que } \\
\text { représentent des réflexes vifs et un clonus. Il y a } \\
\text { un problème neurologique mais je ne peux le } \\
\text { résoudre. }\end{array}$ \\
\hline Dispersé & $\begin{array}{l}\text { Il y a quelques abstractions mais } \\
\text { les hypothèses diagnostiques ne } \\
\text { se réferent pas aux données } \\
\text { cliniques et sont listées de } \\
\text { manière statique, sans être com- } \\
\text { parées et contrastées les unes } \\
\text { aux autres. }\end{array}$ & $\begin{array}{l}\text { Atteinte des membres supérieurs et inférieurs... } \\
\text { Il y a donc atteinte des extrémités. On peut évo- } \\
\text { quer l'alcoolisme, un déficit en vitamine B12, } \\
\text { une polyneuropathie. }\end{array}$ \\
\hline Élaboré & $\begin{array}{l}\text { Les abstractions sont nombreuses } \\
\text { et sont utilisées à bon escient } \\
\text { pour comparer et contraster } \\
\text { des hypothèses diagnostiques. }\end{array}$ & $\begin{array}{l}\text { Il s'agit d'un homme âgé avec apparition } \\
\text { progressive d'un problème sensoriel et moteur } \\
\text { bilatéral et asymétrique. Il y a un déficit moteur } \\
\text { aux membres supérieurs et un syndrome pyramidal } \\
\text { aux membres inférieurs. Je peux donc écarter un } \\
\text { problème périphérique aux membres inférieurs. } \\
\text { Une cause centrale est la plus probable [...] } \\
\text { avec une arthrose cervicale causant une } \\
\text { myélopathie au niveau C8-T1 et une } \\
\text { radiculopathie bilatérale. }\end{array}$ \\
\hline Compilé & $\begin{array}{l}\text { Le clinicien reconnaît d'emblée } \\
\text { un ensemble de données } \\
\text { cliniques qu'il associe à une } \\
\text { hypothèse diagnostique. }\end{array}$ & $\begin{array}{l}\text { C'est un tableau qui me fait penser à un syndrome } \\
\text { sous-lésionnel secondaire à une myélopathie } \\
\text { cervicale causant également une radiculopathie } \\
\text { C8 bilatérale. Il est surprenant que le malade ne } \\
\text { se plaigne pas de douleurs cervicales. }\end{array}$ \\
\hline
\end{tabular}


Figure 2 :

\section{Transformation sémantique et évaluation des hypothèses diagnostiques par comparaison et contraste}

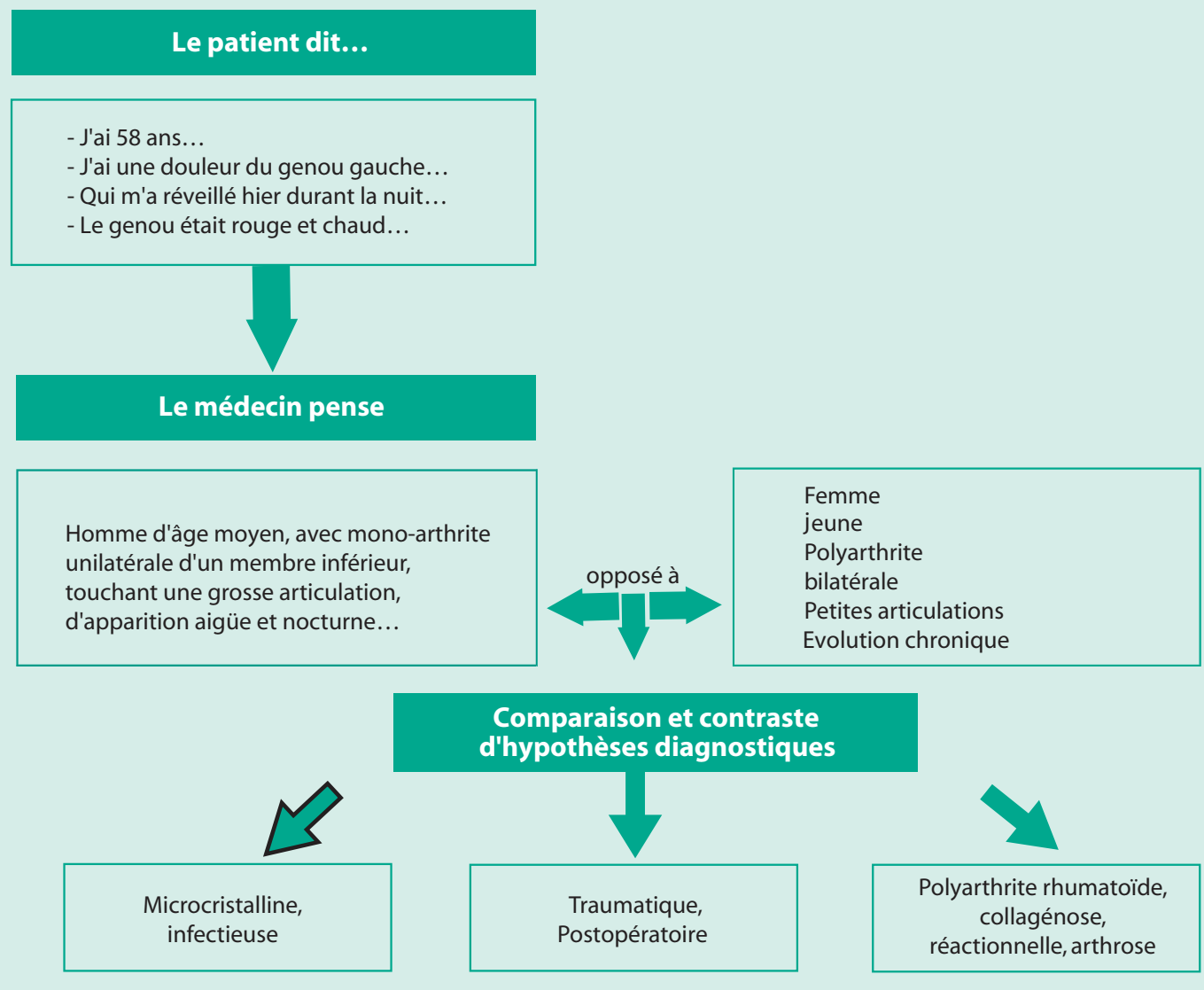

matique; 2) leur utilisation consciente permet de les utiliser de façon stratégique pour confirmer ou infirmer les hypothèses correspondantes ; 3) ils permettent de guider la sélection de l'information, sa mémorisation et son interprétation ${ }^{43}$. Comme tout réseau de connaissances, les scripts évoluent avec l'expérience. Plus le sujet acquiert de l'expérience, plus ses scripts s'enrichissent et plus ils deviennent fonctionnels.

Le savoir biomédical (les sciences de base) demeure néanmoins accessible même s'il est moins présent dans le raisonnement (il s' " encapsule " selon Schmidt ${ }^{28}$ ). Ce savoir place des contraintes sur les valeurs acceptables des signes et de leurs interrelations dans une maladie donnée ${ }^{43,52}$. Il alerte également le clinicien sur des indices ou des séquences d'événements qui violent les attentes physiologiques normales, servant ainsi de critère de cohérence pour les hypothèses émises à propos d'un $\mathrm{Cas}^{53}$. Enfin, les cliniciens l'utilisent dans les situations cliniques où ils ne trouvent pas d'hypothèse pertinente pour comprendre la situation et pour progresser vers la solution du problème à travers une chaîne de raisonnement de cause à effets.

\section{Les schémas}

Certains auteurs proposent une organisation de certaines connaissances en schémas prenant une forme algorithmique dans laquelle un problème clinique spécifique est associé à l'information à rechercher et aux hypothèses diagnostiques à évoquer ${ }^{33,54}$. Par exemple, en cas de dysphagie, le premier embranchement consisterait à distinguer entre une origine oropharyngée ou œesophagienne, 


\section{Références}

ensuite à déterminer une étiologie mécanique ou neuromusculaire et finalement à tester les diverses entités diagnostiques propres à chaque catégorie. L'utilisation de ces schémas et la reconnaissance automatique d'une configuration de signes (pattern recognition) ont été associées à une meilleure performance diagnostique ${ }^{33}$.

\section{La démarche clinique et le recueil des données}

Une fois les processus de raisonnement et les modèles d'organisation des connaissances définis, voyons maintenant comment se concrétise la résolution de cas cliniques, en s'intéressant à quelques-unes de ses particularités, de même qu'au recueil des données cliniques.

\section{La spécificité de cas}

En démontrant que le processus de raisonnement hypothético-déductif est largement utilisé, les travaux d'Elstein et coll. ${ }^{10}$ ont également révélé que la compétence à diagnostiquer varie d'un cas à l'autre pour un même clinicien et qu'il n'existe pas de compétence générale à résoudre les problèmes cliniques. La performance d'un clinicien face à un cas ne permet pas de prédire sa performance face à un autre cas et ce, même au sein de la même discipline. Ceci signifie que, par exemple, la compétence à résoudre un cas de cardiologie ne permet pas de prévoir la performance sur un autre cas de cardiologie. Cette notion, appelée spécificité de cas ou spécificité de conte$\mathrm{nu}$, a de grandes implications en matière d'évaluation de la compétence en raisonnement clinique ${ }^{55}$. Signalons dès maintenant que cela condamne la pratique des oraux longs où la compétence d'un candidat est jugée à partir de son raisonnement sur un seul cas clinique. La recherche nous enseigne qu'il est plus fidèle d'interroger plus brièvement le candidat sur plusieurs cas.

\section{La représentation du problème}

Barrows ${ }^{15}$ a révélé dans ses travaux que si l'on demande aux sujets d'interrompre leur démarche clinique et de formuler en deux ou trois phrases l'essentiel de la situation clinique, les cliniciens expérimentés sont capables de le faire à tout moment alors que les novices en sont incapables. Il s'agit là d'une qualité cruciale, associée au succès diagnostique, qui témoigne de la capacité du clinicien à transformer les données factuelles en constructions schématiques ou sémantiques et à en avoir une vue synthétique, ce qui lui permet de contrôler sa progression vers la solution du problème. Cela permet également de passer d'un problème mal structuré, comportant de multiples données potentielles en un problème gérable, mieux structuré. L'importance d'une capacité à se représenter clairement le problème clinique présenté par le patient est illustrée dans l'étude de Chang et coll ${ }^{56}$. concernant 19 étudiants en fin de cursus et quatre experts examinant un patient standardisé souffrant d'un problème rhumatologique. Les sujets arrivant au bon diagnostic ont recherché et transformé de manière sémantique ${ }^{49}$ trois à quatre fois plus d'informations visant à caractériser la plainte principale. De plus, cette caractérisation (représentation mentale) s'est faite précocement, au moment de la première évocation du diagnostic final. Les auteurs concluent que la compétence diagnostique passe par une représentation pertinente et précoce dans l'esprit du médecin du problème présenté par le patient

\section{La variabilité dans le cheminement du raisonnement}

Plusieurs travaux ${ }^{10,15,53,57,58}$ ont révélé que les cliniciens ne suivent pas tous le même cheminement dans leur progression vers la solution. Même si les recoupements sont importants, ils ne collectent pas toujours les mêmes données et on ne peut déterminer un cheminement commun qui caractériserait l'expertise. Le clinicien efficace ne génère pas plus d'hypothèses, n'en entretient pas plus en mémoire de travail et ne collecte pas plus d'informations cliniques que les novices. En revanche, il se distingue par la génération d'hypothèses de meilleure qualité, par la capacité à recueillir les données cruciales et par une meilleure interprétation des données pour tester ses hypothèses.

La collecte des données cliniques differe d'un clinicien à l'autre ${ }^{53,59}$. Une étude récente ${ }^{59}$ a montré que 20 à $25 \%$ seulement des données collectées et des hypothèses générées sont communes à un groupe d'experts en médecine interne prenant en charge les mêmes patients à partir de leur plainte principale. Chaque médecin possède son propre style de recueil de données. Certains préferent poser plus de questions, explorer plus de systèmes et évaluer immédiatement toute hypothèse diagnostique plausible avant de revenir à leur fil conducteur de raisonne- 
ment; d'autres utilisent une approche plus linéaire, étape par étape, faisant intervenir moins de voies d'exploration. Malgré ces différences, on trouve cependant certaines caractéristiques communes qui sont associées au succès diagnostique ${ }^{14,15}$. Une étude analysant les enregistrements vidéo d'étudiants résolvant le même cas ${ }^{60}$ révèle que quatre comportements de collecte de données sont associés au succès diagnostique. Il s'agit de: 1) la caractérisation détaillée de la plainte principale; 2) la poursuite d'une même ligne de raisonnement dans la récolte de données; 3) la vérification et clarification de l'information et 4) la synthèse de l'information obtenue. Dans une étude concernant six internistes expérimentés confrontés à sept raisons principales de consultation lors d'une simulation de rencontre médicale avec un patient standardisé ${ }^{11}$, les caractéristiques associées au succès diagnostique étaient les suivantes: 1) les questions clés sont posées précocement; 2) les plaintes du patient sont clarifiées; 3) les informations recueillies sont résumées et synthétisées ; 4) le diagnostic final est généré et évalué précocement (Tableau 2).

Ceci a d'importantes implications en enseignement et évaluation. Avant ces travaux, on pensait qu'il existait une compétence générale à bien raisonner sur le plan clinique et que cette compétence générale pouvait s'enseigner et s'évaluer. La méthode d'évaluation appelée PMP (patient management problem) ${ }^{62}$ reposait sur ce postulat et a été décrite en détail, avec ses limites psychométriques, dans un autre article de Pédagogie
Médicale ${ }^{55}$. Les nombreuses recherches ultérieures ont montré qu'il était, cependant, illusoire de chercher à enseigner et à évaluer, pour elle-même, une stratégie unique et optimale de raisonnement clinique.

\section{L'apprentissage et l'enseignement du raisonnement clinique}

Dans le modèle traditionnel de l'enseignement médical les étudiants sont supposés apprendre le raisonnement clinique en lisant des ouvrages de référence, en écoutant des conférences de cas cliniques, en observant des cliniciens expérimentés et en découvrant le processus du raisonnement clinique efficace par essais et erreurs. Ces différentes approches ont chacune leur légitimité et elles sont très complémentaires, à condition d'être articulées les unes avec les autres. La seule lecture de textes de références n'explique pas comment générer des hypothèses diagnostiques pertinentes, comment cheminer efficacement au sein d'une large constellation de signes chez un patient ou comment élucider un problème diagnostique qui se présente de manière atypique. Les conférences de présentation de cas cliniques comportent, elles aussi, des limites substantielles pour l'apprentissage car le processus diagnostique a été réordonné par le présentateur et la logique de l'investigation demeure souvent implicite et imperceptible par les étudiants en médecine. Lorsque débute la partie discussion, a priori favorable à l'apprentissage, tous les détails du cas ont été présentés, de sorte que les étapes

\begin{tabular}{|c|c|}
\hline \multicolumn{2}{|c|}{$\begin{array}{l}\text { Tableau } 2 \text { : } \\
\text { Caractéristiques définissant le style propre de chaque médecin expérimenté } \\
\text { et les stratégies communes dans la collection de données cliniques. }{ }^{61}\end{array}$} \\
\hline Caractéristiques différentes entre médecins & Caractéristiques communes aux médecins \\
\hline - Quantité d'information recueillie & - Questions-clés posées précocement \\
\hline - Buts de l'information recherchée & - Clarification fréquente des plaintes du patient \\
\hline - Stratégies de progression durant la consultation & $\begin{array}{l}\text { - Résumé et synthèse fréquents des informations } \\
\text { recueillies }\end{array}$ \\
\hline - Types d'hypothèses diagnostiques de travail générées & $\begin{array}{l}\text { - Récolte de données ciblées sur des hypothèses } \\
\text { diagnostiques pertinentes } \\
\text { - Diagnostic final généré et évalué précocement }\end{array}$ \\
\hline
\end{tabular}




\section{Références}

intermédiaires par lesquelles les hypothèses diagnostiques ont été éliminées ou confirmées ne sont pas accessibles à l'étudiant ${ }^{63}$. Par ailleurs, les éléments cliniques sont d'emblée présentés et sélectionnés, ce qui ne permet pas à l'auditeur de récolter lui-même ces données, selon son propre chemin de raisonnement ${ }^{21}$. L'observation de cliniciens expérimentés en action comporte également de nombreuses limites si ces derniers ne rendent pas explicites à la fois leurs processus de raisonnement et les connaissances sur lesquelles ils s'appuient pour les développer. Enfin, l'apprentissage de type expérientiel par essais et erreurs peut être stérile si une rétro-action explicite par un enseignant clinicien expérimenté n'est pas apportée, à l'égard à la fois des stratégies développées et des connaissances mobilisées. Plusieurs principes développés dans le cadre de la psychologie cognitive peuvent être exploités pour mettre en ouvre des activités efficaces d'enseignement et d'apprentissage du raisonnement clinique ${ }^{7,64}$. Il est utile de les rappeler avant de les développer:

- établir des liens avec les connaissances antérieures;

- faciliter le raisonnement hypothético-déductif;

- favoriser l'usage à la fois des processus analytique et non analytique de raisonnement clinique;

- favoriser le transfert des connaissances;

- favoriser l'organisation et l'activation des connaissances;

- favoriser une récolte de données cliniques pertinentes et discriminantes.

\section{Établir des liens avec les connaissances antérieures}

La connaissance ne se reçoit pas, elle se construit. Tout apprentissage, qu'il s'agisse d'acquisition de connaissances ou d'habiletés, est le résultat d'une démarche mentale active au cours de laquelle chaque apprenant confronte toute information nouvelle avec ses connaissances antérieures avant de l'incorporer dans sa base de connaissances. Ceci souligne l'importance de la réactivation des connaissances antérieures dans toute situation d'apprentissage.

L'information nouvelle n'est pas assimilée telle quelle. Elle est transformée, mise en relation avec les connaissances antérieures et utilisée pour bâtir une interprétation cohérente de la situation dans laquelle évolue l'individu. Dans une démarche visant à donner un sens aux données, la base de connaissances de l'apprenant permet de sélectionner l'information et de la mémoriser en l'intégrant dans des réseaux de connaissances préexistantes.

Par ailleurs, pour faciliter l'apprentissage de principes ou de concepts d'application générale, il est reconnu qu'il est efficace de placer les apprenants successivement dans plusieurs contextes précis, qui posent problème, ce qui facilite compréhension et application. C'est dire que le raisonnement clinique ne s'apprend pas en termes abstraits. Il s'acquiert par des activités de résolution de problèmes portant sur des cas cliniques particuliers. Nous savons également que les étudiants ne transferent pas spontanément d'un cas à l'autre ce qu'ils ont acquis. Le transfert doit être aidé par des interventions pédagogiques délibérées de la part des enseignants, en présentant aux étudiants plusieurs exemples d'une même entité et en soulignant les éléments similaires et distincts.

La qualité et la richesse de la base de connaissances sont des caractéristiques essentielles de la compétence à raisonner dans un domaine particulier. Les connaissances doivent être élaborées et organisées. Il est bien démontré que plus on permet aux apprenants de créer des liens entre les concepts qu'ils acquièrent et les concepts qu'ils possèdent déjà et que plus on leur permet d'organiser les connaissances en fonction des tâches à réaliser ultérieurement, plus il y a de chance que les connaissances nouvellement apprises puissent être retenues et réutilisées. Soulignons enfin que chaque apprenant construit lui-même sa propre base de connaissances et qu'il est important de favoriser la validation des connaissances nouvellement acquises et des stratégies de raisonnement par une interaction avec les pairs étudiants et avec les cliniciens expérimentés.

\section{Faciliter le raisonnement bypothético- déductif}

En réaction à la tradition qui demandait aux étudiants de recueillir toutes les informations cliniques d'un patient avant d'émettre des hypothèses et de les évaluer, Kassirer ${ }^{63}$ a recommandé d'enseigner aux étudiants les modalités de raisonnement telles que les pratiquent les cliniciens expérimentés et donc de leur enseigner à générer rapidement des hypothèses (selon des processus analytiques et non analytiques, décrits plus haut et rappelés ci-dessous) et à réaliser ensuite une collecte de données orientée. Son approche, adaptée aux caractéristiques de l'enseignement en milieu clinique, est destinée à favoriser les apprentissages nécessaires aux tâches cognitives essentielles de la résolution des problèmes diagnostiques ou thérapeutiques et à éviter les inconvénients du raisonnement en présence du patient, avec toutes les inquiétudes que peut générer chez celui-ci la génération de toutes les hypothèses pos- 
sibles. Pratiquement, les apprenants se réunissent en un groupe de taille modérée sous la supervision d'un animateur. Un de leurs membres connaissant le dossier du patient répond aux questions de ses pairs. Cependant, avant que la réponse à une question ne soit donnée, le groupe doit s'entendre sur le bien-fondé, la pertinence et le but de la demande. L'information donnée est ensuite intégrée dans la démarche diagnostique et le devenir des hypothèses diagnostiques entretenues est discuté. Ce processus peut s'appliquer à la recherche des données cliniques (histoire et examen physique) mais peut également concerner des examens de laboratoires ou des éléments de prise en charge thérapeutique.

Chamberland ${ }^{65}$, à partir de la proposition de Kassirer et des méthodes d'apprentissage par problème décrites par Barrows $^{66}$, a développé une approche systématisée pour soutenir l'apprentissage du raisonnement clinique: les sessions d'ARC (Apprentissage du Raisonnement Clinique). Au sein d'un petit groupe d'étudiants encadrés par un clinicien expérimenté, un étudiant aura au préalable évalué un patient ou étudié un cas et connaît toutes les données cliniques le concernant. Il va jouer le rôle de source des données cliniques du patient. Après avoir donné l'âge, le sexe et la raison principale de consultation, il va se mettre à la disposition du groupe et fournir l'information à la demande explicite des autres membres du groupe. Tout participant peut poser les questions qui vont produire de nouvelles données mais tout étudiant qui pose une question doit la justifier. A cet égard, le moniteur clinicien peut intervenir par des questions telles que: quelles hypothèses diagnostiques avez-vous à l'esprit? Pourquoi posez-vous cette question? Qu'espérez-vous apprendre de la réponse? Une fois la réponse obtenue, celui qui a posé la question doit dès lors interpréter l'information recueillie. L'hypothèse de travail est-elle modifiée ? Le diagnostic estil mieux précisé, d'autres hypothèses sont-elles générées? La session est ainsi conduite selon la séquence d'un entretien clinique habituel, allant de l'interrogatoire à la mise en œuvre d'un plan d'investigation et de traitement.

L'approche permet aux étudiants d'apprendre à avoir une stratégie efficace (recourant à plusieurs processus cognitifs) pour révéler les données importantes et à les interpréter au fur et à mesure de leur découverte. Elle permet également d'exposer les étapes intermédiaires du processus de raisonnement, avec l'aide d'un enseignant qui peut commenter la génération d'une hypothèse incorrecte, demander de poursuivre en profondeur l'exploration d'un symptôme ou suggérer des pistes d'investigation. Elle est structurée par des étapes cruciales telles que la formulation du problème (l'étudiant-interviewer doit décrire en une ou deux phrases l'essentiel de la situation clinique), l'évaluation itérative de la pertinence des hypothèses émises ou la synthèse finale qui permet de souligner les points-clés de la stratégie suivie et de préparer le transfert des apprentissages à d'autres cas cliniques.

Les sessions d'ARC ${ }^{65}$, désormais largement utilisées, permettent, outre le développement du raisonnement clinique, de promouvoir l'acquisition de connaissances nouvelles et de pallier l'imprévisibilité et l'irrégularité des cas auxquels sont exposés les étudiants. Les séances, données tout au long du curriculum clinique, offrent de multiples occasions de raisonnement à voix haute, avec validation de celui-ci par les pairs étudiants et par un enseignant clinicien. Celui-ci, en accord avec les prescriptions de la psychologie cognitive, se met au service des étudiants pour les écouter raisonner à voix haute et guider leur développement du raisonnement et des connaissances en matière diagnostique et thérapeutique.

La méthode suggérée par Kassirer, tout comme l'ARC, répond aux principes pédagogiques rappelés ci-dessus. Les apprenants traitent activement l'information clinique, les connaissances antérieures sont activées, l'apprentissage des principes généraux s'effectue dans le contexte de situations cliniques spécifiques et les connaissances nouvellement acquises sont enrichies de liens fonctionnels et organisées grâce aux discussions et aux échanges entre pairs encadrés par un clinicien expérimenté.

\section{Favoriser à la fois l'usage des processus analytiques et non analytiques de raisonnement clinique}

Les données de recherche sur le raisonnement clinique suggèrent qu'aucune des théories et qu'aucun des modèles présentés ci-dessus et considérés individuellement n'explique parfaitement le raisonnement des cliniciens. Dans certains cas, c'est une approche non analytique (pattern recognition, rappel d' instances) qui mènera à une solution ou mettra sur la piste d'une solution. Dans d'autres cas, c'est une approche analytique (algorithme diagnostique, utilisation des scripts, application du savoir biomédical) qui s'avérera efficace. Les deux approches peuvent même être sollicitées lors d'une même situation clinique. Eva ${ }^{39}$ a montré que la 


\section{Références}

consigne donnée aux cliniciens de faire confiance aux jugements de similarité (processus non analytique), tout en examinant explicitement les données présentées (processus analytique) aboutit à une meilleure performance diagnostique que la consigne de ne faire appel qu'à l'un ou l'autre des deux processus. Une bonne approche pédagogique devra donc promouvoir les deux types de raisonnement dès le début de la formation médicale. Ce genre d'approche aidera les étudiants à se construire un répertoire de stratégies plus éclectique, leur permettant de s'adapter aux besoins de chaque situation particulière.

\section{Favoriser le transfert des connaissances}

Il est important, en matière d'enseignement, de multiplier les exemples, afin que les étudiants accumulent un vaste répertoire de cas pour développer un raisonnement non analytique solide en créant plusieurs voies d'accès en mémoire. L'efficacité de cette approche est optimale lorsque les étudiants, lors de leur apprentissage, sont exposés à plusieurs exemples qui couvrent la gamme des différentes formes sous lesquelles peut se présenter une catégorie diagnostique ${ }^{67}$. Lors de l'apprentissage à base d'exemples, l'enseignant ne devrait pas s'attendre à ce que les étudiants puissent faire, par eux-mêmes, des comparaisons et des liens entre divers problèmes. L'apprentissage du raisonnement par analogie chez les novices est optimisé lorsqu'ils reçoivent des instructions explicites de rechercher les similarités et les concepts partagés par divers problèmes en apparence très différents les uns des autres ou, à l'inverse, d'identifier les spécificités qui distinguent des problèmes en apparence similaires. De plus, lors de la présentation de nouveaux problèmes, les principes et concepts sous-jacents devraient être reliés aux exemples et principes déjà appris antérieurement par les étudiants.

Pour faciliter la coordination des processus analytiques et non analytiques, l'approche pédagogique traditionnelle, recommandant une séquence initiale de formation dédiée aux connaissances biomédicales suivie d'une séquence de formation pratique en contexte clinique, est confrontée à plusieurs limites. Il sera plus judicieux d'y substituer un dispositif qui intègre simultanément les approches analytiques et non analytiques tout au long de l'apprentissage médical et qui pourrait favoriser une utilisation pertinente des deux processus ${ }^{39}$.

Enfin, l'apprentissage des connaissances utiles à la démarche diagnostique devrait aussi prendre en compte la nécessité que les représentations cognitives concernées soient facilement disponibles lors d'un entretien clinique. Lorsque les étudiants s'exercent au recueil de données tout en connaissant à l'avance le diagnostic ou le thème d'une session d'apprentissage, ils sont beaucoup moins en mesure de déterminer par eux-mêmes s'ils seraient capables de reconnaître la présentation clinique hors d'un tel contexte. En fait, Hatala ${ }^{68}$ a montré que des exercices utilisant un répertoire varié de situations différentes conduisent à un meilleur apprentissage et induisent une plus grande capacité de reconnaître des nouveaux cas que s'ils exploitent des catégories diagnostiques prédéterminées.

\section{Favoriser l'organisation et l'activation des connaissances}

La psychologie cognitive met l'accent sur l'importance de disposer de connaissances organisées et construites dans le contexte de cas spécifiques, tout en faisant référence aux connaissances antérieures ${ }^{7}$. La catégorisation des pathologies à l'aide d'exemples prototypiques facilite l'organisation efficace des connaissances. Pour aider l'étudiant à construire des connaissances utilisables en pratique, il est préférable d'utiliser un nombre limité de prototypes diagnostiques par catégorie de maladie ${ }^{69}$. Par exemple, pour la catégorie "douleur rétrosternale ", on pourrait utiliser les prototypes "infarctus myocardique ", " péricardite ", " dissection aortique " et " reflux gastroœsophagien ». Ces prototypes devraient de plus être à un niveau intermédiaire de spécificité pour être mieux retenus: par exemple "infarctus du myocarde " plutôt qu' " athérosclérose ", trop général, ou que " vasculite coronarienne ", trop spécifique. Une fois ces prototypes organisés en mémoire, et à ce moment-là seulement, des pathologies plus spécifiques ou plus générales pourront être ancrées sur cette colonne vertébrale de prototypes de spécificité intermédiaire, pour étendre le réseau de connaissances avec tous les liens nécessaires. Pour caricaturer, il n'est, en effet, pas très utile à l'étudiant d'entendre son précepteur évoquer une porphyrie face à une douleur abdominale s'il n'a jamais entendu parler d'appendicite...

Pour amener les étudiants à activer leurs connaissances face à une situation clinique, il faut les aider à mieux comprendre les structures profondes d'un problème et à en construire une représentation adéquate. Pour ce faire, Bordage et coll. proposent de rendre plus visibles les associations sémantiques, que ce soit lors de l'enseignement ${ }^{48}$ ou dans les livres de références ${ }^{70}$. Mandin et 
coll. proposent de favoriser la construction d'autres formes de représentation mentale, en reconnaissant quel schéma est adapté à la situation clinique, de manière à résoudre le cas de façon plus logique et organisée ${ }^{54}$. Cette théorie ( $c f$. section sur l'organisation des connaissances) a servi de base à la construction d'un curriculum complet à l'Université de Calgary, dont on attend les résultats d'une évaluation future ${ }^{71}$. Dans un registre similaire, plusieurs méthodes d'apprentissage proposent l'utilisation de la schématisation pour favoriser l'organisation et l'activation des connaissances. Ainsi en est-il de l'approche par construction de cartes conceptuelles récemment exposées dans ce journal ${ }^{72,73}$.

Un travail récent a testé en pratique les effets d'une intervention pédagogique s'appuyant sur la transformation sémantique ${ }^{74}$. Cette intervention courte avait pour but d'aider les étudiants à dépasser une approche fragmentaire basée sur des symptômes isolés, pour utiliser une stratégie de résolution de problème plus riche, en transformant les symptômes et signes en représentations sémantiques, permettant ainsi de mieux comparer et contraster des hypothèses diagnostiques. Elle a permis une meilleure utilisation de la transformation sémantique mais ne s'est pas traduite par une meilleure interprétation des données et une meilleure performance diagnostique. Ceci reflète, une fois de plus, la nécessité de développer simultanément la base de connaissances face à un problème, la représentation globale des ces connaissances et l'application de stratégies variées de raisonnement.

Finalement, il faut avoir conscience que les médecins expérimentés ont un savoir compilé et une tendance à utiliser des raccourcis de la pensée lors de leur raisonnement; de ce fait, les apprenants peuvent avoir des difficultés à les suivre dans leur démarche de raisonnement clinique et à tirer tous les bénéfices de leur observation $^{75}$. Il devient dès lors important pour le précepteur de prendre la peine de dérouler le fil de son raisonnement et de rendre explicite tous les liens nécessaires entre la situation présente, la physiopathologie et les cas cliniques précédemment rencontrés, dans le but de clarifier la nature de la relation unissant tel signe ou symptôme et une entité diagnostique spécifique.

\section{Favoriser une récolte de données cliniques pertinentes et discriminantes}

Diverses études effectuées pour des données visuelles ${ }^{31,37,76}$ et non visuelles ${ }^{61}$ ont mis en évidence diverses caractéristiques conduisant à une meilleure acquisition de données cliniques. Ces travaux confirment l'importance de cibler le recueil d'informations en fonction des hypothèses diagnostiques évoquées, dont la pertinence va influencer la qualité de l'information recueillie. Ainsi, de la part de l'enseignant clinicien, favoriser l'exhaustivité des données pour elle-même n'est pas une stratégie pédagogique qui favorise la pertinence du recueil d'informations permettant un raisonnement de qualité. Il est plus adéquat d'encourager l'étudiant à justifier les données recueillies, ce qui complète bien les principes d'enseignement du raisonnement clinique énoncés par Kassirer. Cette approche n'empêche pas l'étudiant, dans un deuxième temps, d'effectuer une revue de systèmes plus exhaustive, mais elle insiste sur l'importance d'initier le recueil de l'information de façon logique en rapport avec la plainte principale.

Étant donné que l'exactitude d'une hypothèse précoce peut influencer la qualité de la récolte subséquente des informations cliniques et leur l'interprétation ${ }^{37,38,76}$, il importe donc d'encourager à considérer les hypothèses qui forment la liste des diagnostics différentiels. En psychologie, ce genre d'approche est efficace pour l'élimination du biais de confirmation, c'est-à-dire la tendance à considérer surtout les informations qui accréditent une seule hypothèse et à négliger celles qui la discréditent ${ }^{77}$. De plus, lors de séquences d'enseignement basées sur des exemples, il est important d'établir explicitement les liens entre une manifestation clinique particulière et une liste de diagnostics possibles et de contraster ces derniers entre eux afin d'aider les étudiants à mieux retenir ce qui les différencie ${ }^{78}$. Il est à noter qu'un diagnostic différentiel n'est pas une simple liste exhaustive des diagnostics que l'on peut trouver dans un manuel de référence en médecine, mais une liste de diagnostics pertinents pour la situation clinique particulière. Une fois encore, une approche prototypique peut aider l'étudiant à générer des diagnostics différentiels pertinents. La recherche explicite d'éléments discriminants, tels que symptômes, signes physiques ou mécanismes physiopathologiques, pour différencier les hypothèses d'un diagnostic différentiel favorisera le développement en mémoire de liens multiples qui serviront éventuellement de voies d'accès aux connaissances face à des cas nouveaux. Ainsi, le fait d'être soulagé par la prise d'aspirine n'est pas un bon élément discriminant entre une arthrose dégénérative et une arthrite rhumatoïde. 


\section{Références}

En revanche, la topographie des articulations touchées ou l'âge du patient le seront davantage. Les étudiants en médecine, et même les cliniciens d'expérience, utilisent trop souvent des éléments non discriminants pour étayer un diagnostic provisoire, souvent unique, phénomène appelé "pseudo-diagnosticité » ${ }^{79} 80$. Wigton a démontré que la recherche de tels éléments discriminants est non seulement bénéfique pour porter un diagnostic juste ${ }^{81}$ mais aussi pour l'apprentissage des étudiants. Lorsque l'enseignant met à jour de façon explicite les éléments discriminants d'une situation clinique donnée, les étudiants les retiendront davantage et les utiliseront mieux ensuite, phénomène appelé rétro-action cognitive par ces auteurs. Leffet d'apprentissage sera d'autant plus bénéfique si l'enseignant note également la probabilité a priori des diagnostics entretenus ${ }^{82-84}$.

Les objectifs de base de l'enseignement du raisonnement clinique et quelques moyens pour les réaliser sont proposés dans le tableau 3.

\section{Conclusion}

Tout au long de cette revue, nous avons essayé de synthétiser certains aspects relatifs au raisonnement clinique et avons tenté de proposer une application de ces connaissances en pratique. Cette démarche, également entreprise par des auteurs anglo-saxons ${ }^{85,86}$, illustre la nécessité de s'appuyer sur des théories et des modèles issus notamment de la psychologie cognitive, pour tenter d'expliquer les mécanismes du raisonnement clinique. Bien que cette recherche ait abouti à plusieurs innovations en éducation médicale, elle doit encore être complétée, notamment par une évaluation de son impact pratique, par exemple, sur le comportement des médecins et la qualité des soins.

Ce domaine ne pourra se développer qu'en collaboration avec des enseignants cliniciens, de manière à traiter des questions de recherche pertinentes. Ceci demande un certain changement de culture dans l'esprit des éducateurs qui pensent volontiers à utiliser les données probantes issues de la recherche et disponibles pour résoudre leurs

\begin{tabular}{|l|l|}
\multicolumn{2}{|c|}{ Principes d'enseignement au lit du malade issus des théories de psychologie cognitive } \\
sur l'organisation des connaissances
\end{tabular}


problèmes cliniques, mais ne font pas forcément la même démarche quand il s'agit d'enseigner ${ }^{87}$. Leur investissement et leur intérêt personnels permettront encore d'améliorer la qualité de la formation des futurs médecins et, ce faisant, on l'espère, la qualité des soins.

\section{Remerciements}

Une partie de cette revue est une mise à jour d'un chapitre écrit pour l'ouvrage: "AF Junod, coll. MR Nendaz. Décision médicale ou la quête de l'explicite. Genève: Editions Médecine et Hygiène, 2003 ». Nous remercions le directeur des Editions Médecine et Hygiène de nous avoir aimablement autorisés à utiliser ce matériau.

\section{Contributions}

Mathieu Nendaz, Bernard Charlin, Vicki LeBlanc et Georges Bordage ont participé collégialement et solidairement à la rédaction initiale du manuscrit et à ses révisions successives. Mathieu Nendaz a en plus assuré la coordination de la version finale.

\section{Figure 3 : \\ Un raisonnement clinique de qualité}

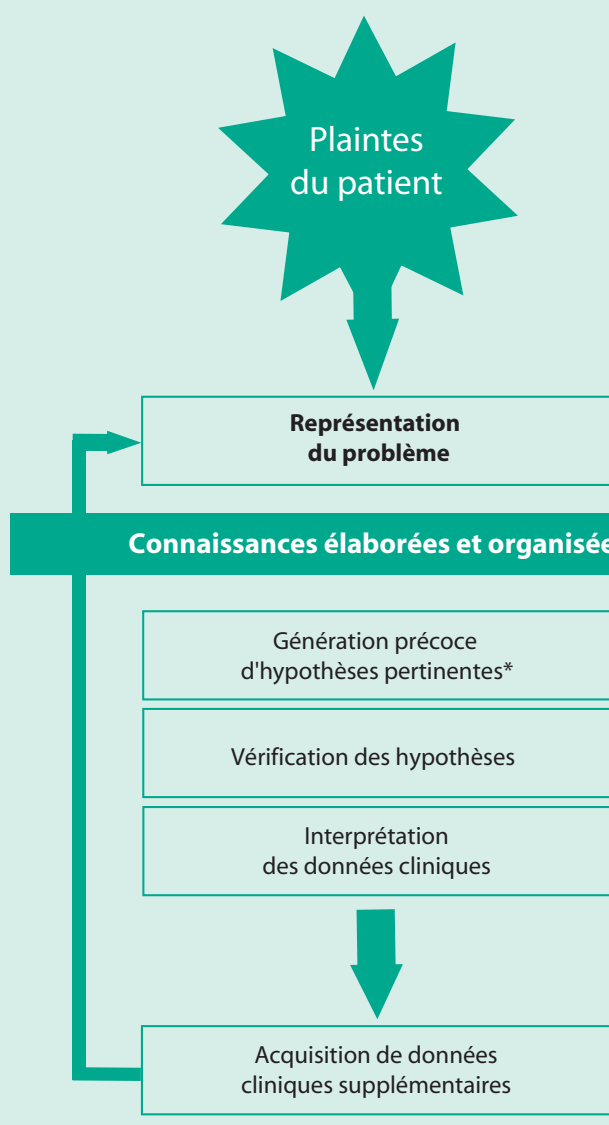

* Peuvent être à divers niveaux de spécificité : générales (problème cardiaque, problème inflammatoire) ou plus spécifiques (péricardite constrictive, lupus érythémateux disséminé), les niveaux intermédiaires de spécificité étant les plus optimaux. 


\section{Références}

\section{Références}

1. Higgs J, Jones M. Clinical reasoning in the health professions. In: Higgs J, Jones, $M$ (Eds). Clinical Reasoning in the Health Professions. 2nd ed. Oxford: Butterworth-Heineman Ltd; 2000:3-14.

2. Harris I. New Expectations for Professional Competence. In: Curry L, Wegin, JF (Eds). Educating Professionals Responding to new expectations for competence and accountability. San Francisco: Jossey-Bath Publishers; 1993:17-52.

3. Newble D, Norman, van der Vleuten C. Assessing Clinical Reasoning. In: Higgs J, Jones $M(E d)$ Clinical Reasoning in the Health Professions. Oxford: Butterworth-Heinemann Ltd, 2000:156-65.

4. Nendaz M. Comment les médecins raisonnent-ils? In: Junod $A(E d)$ Décision médicale ou la quête de l'explicite. Genève: Médecine \& Hygiène, 2004:15-30.

5. Wilkerson L, Irby DM. Strategies for improving teaching practices: a comprehensive approach to faculty development. Acad Med 1998; 73:387-96.

6. Irby DM. Shifting paradigms of research in medical education. Acad Med 1990;65:622-3.

7. Regehr G, Norman GR. Issues in cognitive psychology: implications for professional education. Acad Med 1996; 71:988-1001.

8. Bernard JL, Reyes P. Apprendre en médecine (1ère partie). Pédagogie Médicale 2001;2:163-9.

9. Junod A. Décision médicale ou la quête de l'explicite. Genève: Médecine et Hygiène; 2004.

10. Elstein AS, Shulman, LS, Sprafka SA. Medical Problem Solving: An Analysis of Clinical Reasoning. Cambridge(MA): Harvard University Press; 1978.

11. Elstein AS, Shulman LS, Sprafka SA. Medical Problem Solving, a Ten-Year Retrospective. Evaluation \& The Health Profession 1990;13:5-36.

12. Norman G, Brooks L, Colle C, Hatala R. The benefit of diagnostic hypotheses in clinical reasoning: Experimental study of an instructional intervention for forward and backward reasoning. Cognition and instruction 1999;17: 433-48.

13. Kassirer JP, Gorry GA. Clinical problem solving: a behavioral analysis. Ann Intern Med 1978;89:245-55.

14. Neufeld VR, Norman GR, Feightner JW, Barrows HS. Clinical problem-solving by medical students: a cross-sectional and longitudinal analysis. Med Educ 1981;15:315-22.

15. Barrows HS, Norman GR, Neufeld VR, Feightner JW. The clinical reasoning of randomly selected physicians in general medical practice. Clin Invest Med 1982;5:49-55.
16. Norman GR, Tugwell P, Feightner JW. A comparison of resident performance on real and simulated patients. JMed Educ 1982;57:708-15.

17. Coughlin LD, Patel VL. Processing of critical information by physicians and medical students. J Med Educ 1987;62:818-28.

18. Patel VL, Groen GJ, Frederiksen CH. Differences between medical students and doctors in memory for clinical cases. Med Educ 1986;20:3-9.

19. Patel VL, Groen GJ, Arocha JF. Medical expertise as a function of task difficulty. Mem Cognit 1990;18:394-406.

20. Eva K, Brooks LR, Norman, GR. Forward reasoning as a hallmark of expertise in medicine: logical, psychological and phenomelogical inconsistencies. In: Shokov $S$ (Ed). Advances in Psychological Research. New York: Nova Science Publishers, Inc, 2002:41-69.

21. Nendaz MR, Raetzo MA, Junod AF, Vu NV. Teaching Diagnostic Skills: Clinical Vignettes or Chief Complaints? Adv Health Sci Educ Theory Pract 2000;5:3-10.

22. Wigton RS. Use of linear models to analyze physicians' decisions. Med Decis Making 1988;8:241-52.

23. Elieson SW, Papa FJ. The effects of various knowledge formats on diagnostic performance. Acad Med 1994;69 (10 Suppl):S81-3.

24. Swanson DB, Feltovich PJ, Johnson PE. Psychological Analysis of Physician Expertise: Implications for Design of Decision Support Systems. In: Shires DB and Wolf (Eds). Proceedings of MEDINFO 77 . Amsterdam (NorthHolland), 1977:161-4

25. Groen GJ, Patel VL. Medical problem-solving: some questionable assumptions. Med Educ 1985;19:95-100.

26. Groen G, Pate, VL. Knowledge-based solutions strategies in medical reasoning. Cognit Sci 1986;10:91-116.

27. Brooks L. Decentralised control in categorisation: the role of prior processing episodes. In: Neisser U (Ed) Concepts and Conceptual Development: Ecological and Intellectual Factors in Categorisation. Cambridge: Cambridge University Press, 1987:141-74.

28. Schmidt HG, Norman GR, Boshuizen HP. A cognitive perspective on medical expertise: theory and implication. Acad Med 1990;65:611-21.

29. Brooks LR, Norman GR, Allen SW. Role of specific similarity in a medical diagnostic task. J Exp Psychol Gen 1991; 120:278-87.

30. Hatala RA, Norman GR, Brooks LR. The effect of clinical history on physicians' ECG interpretation skills. Acad Med 1996;71(10 Suppl):S68-70. 
31. Brooks LR, LeBlanc, V.R., Norman, G.R. On the difficulty of noticing obvious features in patient appearance. Psychol Sci 2000;11:112-7.

32. Eva KW, Brooks LR. The under-weighting of implicitly generated diagnoses. Acad Med 2000;75 (10 Suppl):S813.

33. Coderre S, Mandin, H., Harasym, P.H., Fick, G. H. Diagnostic reasoning strategies and diagnostic success. Med Educ 2003;37:695-703.

34. Kulatunga-Moruzi C, Brooks LR, Norman GR. Coordination of analytic and similarity-based processing strategies and expertise in dermatological diagnosis. Teach Learn Med 2001;13:110-6.

35. Norman GR. From theory to application and back again: Implications of research on medical expertise for psychological theory. Canadian Journal of Experimental Psychology 2005:59:35-40.

36. Norman GR, Brooks LR, Regehr G, Marriott M, Shali V. Impact of feature interpretation on medical student diagnostic performance. Acad Med 1996;71(1 Suppl): S108-9.

37. LeBlanc V, Norman $G$, Brooks L. Effect of a diagnostic suggestion on diagnostic accuracy and clinical feature identification. Acad Med 2001;76 (Suppl 10):S18-S20.

38. LeBlanc VR, Dore K, Norman GR, Brooks LR. Limiting the playing field: does restricting the number of possible diagnoses reduce errors due to diagnosis-specific feature identification? Med Educ 2004;38:17-24.

39. Eva $K W$. What every teacher needs to know about clinical reasoning. Med Educ 2005;39:98-106.

40. Bordage G. Elaborated knowledge: a key to successful diagnostic thinking. Acad Med 1994;69: 883-5.

41. Grant J, Marsden P. The Structure of Memorized Knowledge in Students and Clinicians: An Explanation for Diagnostic Expertise. Medical Education 1987;21:928.

42. Kahneman D, Miller KT. Norm theory: Comparing reality to its alternatives. Psychological Review 1986;93:13653.

43. Charlin B, TardifJ, Boshuizen HPA. Scripts and Medical Diagnostic Knowledge: Theory and Applications for Clinical Reasoning Instruction and Research. Academic Medicine 2000;75:182-90.

44. Custers EJFM, Regehr G, Norman GR. Mental representations of medical diagnostic knowledge: A review. Acad Med 1996;71(10 suppl):S55-61.

45. Allen SW, Norman GR, Brooks LR. Experimental studies of learning dermatological diagnosis: the impact of examples. Teach Learn Med 1992;4:35-44.
46. Bordage $G$, Zacks $R$. The structure of medical knowledge in the memories of medical students and general practitioners: categories and prototypes. Med Educ 1984;18:406-16.

47. Papa FJ, Stone RC, Aldrich DG. Further evidence of the relationship between case typicality and diagnostic performance: implications for medical education. Acad Med 1996;71(1 Suppl):S10-2.

48. Lemieux $M$, Bordage G. Propositional vs Structural Semantic Analyses of Medical Diagnostic Thinking. Cognitive Science 1992;16:185-204.

49. Bordage G, Connell KJ, Chang RW, Gecht MR, Sinacore $J M$. Assessing the semantic content of clinical case presentations: studies of reliability and concurrent validity. Acad Med 1997;72(10 Suppl 1):S37-9.

50. Nelson K. Event knowledge and cognitive development. In: Nelson K, ed. Event Knowledge: Structure and Function in Development. Hillsdale, NJ: Lawrence Erlbaum Associates; 1986.

51. Fayol M, Monteil J-M. The notion of script: from general to developmental and social psychology. Eur Bull Cogn Psychol 1988;8:335-61.

52. Feltovich PJ, Barrows, H.S. Issues of Generality in Medical Problem Solving. In: Schmidt H.G. De Volder ML(Eds). Tutorials in Problem-Based Learning: A new Direction in Teaching the Health Professions. Assen(Holland): Van Gorcum, 1984:128-42.

53. Kassirer JP. Diagnostic reasoning. Ann Intern Med 1989;110:893-900.

54. Mandin H, Jones A, Woloschuk W, Harasym P. Helping students learn to think like experts when solving clinical problems. Acad Med 1997;72:173-9.

55. Charlin B, Bordage G, Van der Vleuten C. L'évaluation du raisonnement clinique. Pédagogie Médicale 2003;4:42-51.

56. Chang RW, Bordage G, Connell KJ. The importance of early problem representation during case presentations. Acad Med 1998;73(10 Suppl):S109-11.

57. Gale J, Marsden P. Clinical problem solving: the beginning of the process. Med Educ 1982;16:22-6.

58. Gale J, Marsden P. The role of the routine clinical history. Med Educ 1984;18:96-100.

59. Nendaz MR, Gut AM, Perrier A, et al. Degree of concurrency among experts in data collection and diagnostic hypothesis generation during clinical encounters. Med Educ 2004;38:25-31.

60. Hasnain M, Bordage G, Connell KJ, Sinacore JM. History-taking behaviors associated with diagnostic competence of clerks: an exploratory study. Acad Med 2001; 76(10 Suppl): S14-7. 


\section{Références}

61. Nendaz MR, Gut AM, Perrier A et al. Common strategies in clinical data collection displayed by experienced clinician-teachers in internal medicine. Med Teach 2005;27: 415-21.

62. McGuire $\mathrm{CH}$, Babbott D. Simulation techniques in the measurement of problem solving skills. J Educ Measur 1967;4:1-10.

63. Kassirer JP. Teaching clinical medicine by iterative hypothesis testing. Let's preach what we practice. $N$ Engl J Med 1983;309:921-3.

64. Tardif J. Pour un enseignement stratégique: l'apport de la psychologie cognitive. Montréal: Les Editions Logiques; 1992.

65. Chamberland $M$. Les séances d'apprentissage au raisonnement clinique $(A R C)$. Un exemple d'activité pédagogique contextualisée adaptée aux stages cliniques en médecine. Ann Med Interne (Paris) 1998;149:479-84.

66. Barrows HS, Tamblyn RM. Problem-Based Learning: An Approach to Medical Education. New York, N.Y: Springer Publishing Co., Series on Medical Education; 1980.

67. Eva KW, Neville AJ, Norman GR. Exploring the etiology of content specificity: factors influencing analogic transfer and problem solving. Acad Med 1998;73(10 Suppl):S1-5.

68. Hatala RM, Brooks LR, Norman GR. Practice makes perfect: the critical role of mixed practice in the acquisition of ECG interpretation skills. Adv Health Sci Educ Theory Pract 2003;8:17-26.

69. Bordage $G$. The curriculum: overloaded and too general? Med Educ 1987;21:183-8.

70. Bordage G, Lemieux $M$. Which medical textbook to read? Emphasizing semantic structures. Acad Med 1990;65 (9 Suppl):S23-4.

71. Mandin H, Harasym P, Eagle C, Watanabe M. Developing a "clinical presentation" curriculum at the University of Calgary. Acad Med 1995;70:186-93.

72. Côté DJ, Bellavance C, Chamberland M, Graillon A. Un programme pour aider les étudiants en médecine à développer leurs stratégies d'apprentissage. Pédagogie Medicale 2005;5:95-102.

73. Marchand C, Gagnayre R. Utilisation de la carte conceptuelle auprès de formateurs en santé pour l'apprentissage de concepts pédagogiques. Pédagogie Medicale 2005;5:13-23.
74. Nendaz MR, Bordage G. Promoting diagnostic problem representation. Med Educ 2002;36:760-6.

75. Nendaz MR, Junod AF, Vu NV, Bordage G. Eliciting and displaying diagnostic reasoning during educational rounds in internal medicine: who learns from whom? Acad Med 1998;73(10 Suppl):S54-6.

76. LeBlanc VR, Brooks LR, Norman GR. Believing is seeing: the influence of a diagnostic hypothesis on the interpretation of clinical features. Acad Med 2002; 77(10 Suppl): S67-9.

77. Klayman J, Brown K. Debias the environment instead of the judge: An alternative approach to reducing error in diagnostic (and other) judgment. Cognition 1993;49:97-122.

78. Avrahami J, Kareev Y, Bogot Y, Caspi R, Dunaevsky S, Lerner S. Learning by examples: Implications for the process of category acquisition. QJ Exp Psychol A 1997;50 A(3):586-606.

79. Friedman MH, Connell KJ, Olthoff AJ, Sinacore JM, Bordage $G$. Medical student errors in making a diagnosis. Acad Med 1998;73(10 Suppl):S19-21.

80. Gruppen LD, Wolf FM, Billi JE. Information gathering and integration as sources of error in diagnostic decision making. Med Decis Making 1991;11:233-9.

81. Wigton RS, Connor JL, Centor RM. Transportability of a decision rule for the diagnosis of streptococcal pharyngitis. Arch Intern Med 1998;146:81-3.

82. Poses RM, Cebul RD, Wigton RS, Centor RM, Collins M, Fleischli $G$. Controlled trial using computerized feedback to improve physicians' diagnostic judgments. Acad Med 1992;67:345-7.

83. Tape TG, Kripal J, Wigton RS. Comparing methods of learning clinical prediction from case simulations. Med Decis Making 1992;12:213-21.

84. Wigton RS, Patil KD, Hoellerich VL. The effect of feedback in learning clinical diagnosis. J Med Educ 1986;61:81622.

85. Norman G. Research in clinical reasoning: past history and current trends. Med Educ 2005;39:418-27.

86. Gruppen LD. Clinical reasoning. In: Norman $G$, van der Vleuten C, Newble D, eds. International Handbook of Research in Medical Education. Boston: Kluwer. 2002.

87. Bligh J. Research in medical education at the start of the century. Med Educ 2002;36:1000-1.

Manuscrit reçu le 15 septembre 2005; commentaires éditoriaux formulés aux auteurs le 20 septembre 2005; accepté pour publication le 29 septembre 2005. 